\title{
Student difficulties with the basics for a system of non-interacting identical particles
}

\author{
Emily Marshman \\ Department of Physics and Astronomy, Community College of Allegheny County, Pittsburgh, PA 15260 \\ Christof Keebaugh \\ Department of Physics and Astronomy, Franklin and Marshall College, Lancaster, PA 17603 \\ Chandralekha Singh \\ Department of Physics and Astronomy, University of Pittsburgh, Pittsburgh, PA 15260
}

\begin{abstract}
We discuss an investigation of upper-level undergraduate and graduate students' difficulties with fundamental concepts involving a system of identical particles. The investigation was carried out in advanced quantum mechanics courses by administering written questions and conducting individual interviews with students. We find that students share many common difficulties related to these quantum mechanical concepts, e.g., with regard to the many-particle Hamiltonian and stationary state wavefunctions for a system of non-interacting identical particles.
\end{abstract}




\section{INTRODUCTION AND BACKGROUND}

There have been a number of research studies aimed at investigating student reasoning in quantum mechanics (QM) and improving student understanding of QM [1-45]. However, there have been relatively few investigations into student difficulties with fundamental concepts involving a system of identical particles. Here we discuss upperlevel undergraduate and graduate student difficulties with these concepts. Our goal is to use these difficulties found via research as resources and develop research-based learning tools. Through researching students' reasoning about a system of identical particles, we found common student difficulties that can hinder their development of a consistent and coherent knowledge structure pertaining to these concepts.

In nature, there are two general types of particles: fermions with a half-integer spin quantum number (e.g., electrons and protons) and bosons with an integer spin quantum number (e.g., photons and mesons). A system of $N$ identical particles consists of $N$ particles of the same type (e.g., electrons). For a system of identical particles in classical mechanics (e.g., five identical tennis balls), each particle can be distinguished from all the other particles. In contrast, in QM, identical particles are indistinguishable and there is no measurement that can be performed to distinguish these particles from one another. For example, if the coordinates of two identical particles are interchanged, there is no physical observable that would reflect this interchange. To reflect the indistinguishability of these identical particles and make the properties of fermions and bosons consistent with observations, the wavefunction for a system of identical fermions must be completely antisymmetric and the wavefunction for a system of identical bosons must be completely symmetric with respect to exchange of two particles. Furthermore, one property that distinguishes these two types of particles is that two or more bosons can occupy the same single-particle state, but two or more fermions can never occupy the same singleparticle state. The restriction for fermions is known as the Pauli exclusion principle and is consistent with a system of identical fermions having a completely antisymmetric wavefunction.

Here we focus on the many-particle Hamiltonian and stationary state wavefunctions that are solutions to the TimeIndependent Schrödinger Equation (TISE) for a system of non-interacting identical particles. Unless otherwise stated, we will refer to the stationary state wavefunction as the wavefunction. For a system of $N$ non-interacting identical particles, the Hilbert space $(\mathscr{H})$ for the many-particle system in $M^{N}$ dimensions is $\mathscr{H}=\mathscr{H}_{1} \otimes \mathscr{H}_{2} \otimes \cdots \otimes \mathscr{H}_{N}$, which is the direct product of the $M$-dimensional Hilbert spaces for each particle $\mathscr{H}_{i}$. The many-particle Hamiltonian for the system of $N$ non-interacting identical particles in the product space is $\hat{\mathbf{H}}=\hat{H}_{1} \otimes \hat{I}_{2} \otimes \hat{I}_{3} \otimes \cdots \otimes \hat{I}_{N}+\hat{I}_{1} \otimes$ $\hat{H}_{2} \otimes \hat{I}_{3} \otimes \cdots \otimes \hat{I}_{N}+\cdots+\hat{I}_{1} \otimes \hat{I}_{2} \otimes \cdots \hat{I}_{N-2} \otimes \hat{H}_{N-1} \otimes$ $\hat{I}_{N}+\hat{I}_{1} \otimes \hat{I}_{2} \otimes \cdots \otimes \hat{I}_{N-1} \otimes \hat{H}_{N}$ where the single-particle
Hamiltonian, $\hat{H}_{i}$, and the identity operator, $\hat{I}_{i}$, for the $i^{t h}$ particle are in the $M$-dimensional Hilbert space $\mathscr{H}_{i}$. We will use the following shorthand notation for the many-particle Hamiltonian $\hat{\mathbf{H}}=\sum_{i} \hat{\mathbf{H}}_{i}=\hat{\mathbf{H}}_{1}+\hat{\mathbf{H}}_{2}+\hat{\mathbf{H}}_{3}+\cdots+\hat{\mathbf{H}}_{N}$ in which $\hat{\mathbf{H}}_{i}=\hat{I}_{1} \otimes \hat{I}_{2} \otimes \cdots \otimes \hat{I}_{i-1} \otimes \hat{H}_{i} \otimes \hat{I}_{i+1} \cdots \otimes \hat{I}_{N}$ is the Hamiltonian of the $i^{\text {th }}$ particle in the $M^{N}$ dimensional product space. Thus, in the rest of this paper, all the boldface Hamiltonians are in the product space.

In order to determine the many-particle stationary state wavefunction for a system of non-interacting identical particles, one must first solve the single-particle TISE. The single-particle stationary state wavefunctions are solutions to the single-particle TISE, i.e., $\hat{H}_{i} \psi_{n_{j}}\left(x_{i}\right)=E_{n_{j}} \psi_{n_{j}}\left(x_{i}\right)$ for the $i^{t h}$ particle in the state $\psi_{n_{j}}\left(x_{i}\right)$. Next, one should construct the many-particle stationary state wavefunction for a system of non-interacting identical particles that can be treated as distinguishable as the product of the single-particle stationary state wavefunctions (these product states can be used as basis states to construct the many-particle stationary state wavefunction for identical fermions or bosons). The final step is to appropriately symmetrize the many-particle stationary state wavefunction for a system of non-interacting identical fermions or bosons using the basis states in the product space.

If we have a system of two non-interacting electrons in one spatial dimension in which one electron is in the single-particle state denoted by $\psi_{n_{1}}$ and the other electron is in the single-particle state denoted by $\psi_{n_{2}}$ $\left(n_{1} \neq n_{2}\right)$, then the wavefunction for the system of two electrons must be completely antisymmetric. Ignoring spin, this normalized two-particle wavefunction is $\Psi\left(x_{1}, x_{2}\right)=\frac{1}{\sqrt{2}}\left[\psi_{n_{1}}\left(x_{1}\right) \psi_{n_{2}}\left(x_{2}\right)-\psi_{n_{2}}\left(x_{1}\right) \psi_{n_{1}}\left(x_{2}\right)\right]$ in which $x_{1}$ denotes the coordinate of the first electron and $x_{2}$ denotes the coordinate of the second electron. This completely antisymmetric wavefunction reflects the fact that one electron is in the single-particle state $\psi_{n_{1}}$ and the other electron is in the single-particle state $\psi_{n_{2}}$, but we cannot say which electron is in which singleparticle state. It is consistent with Pauli's exclusion principle. For example, for a system of two fermions both in the same single-particle state $\psi_{n_{1}}$, the antisymmetric wavefunction (ignoring the spin) would be $\Psi\left(x_{1}, x_{2}\right)=$ $\frac{1}{\sqrt{2}}\left[\psi_{n_{1}}\left(x_{1}\right) \psi_{n_{1}}\left(x_{2}\right)-\psi_{n_{1}}\left(x_{1}\right) \psi_{n_{1}}\left(x_{2}\right)\right]=0$. Thus, there is no wavefunction for a system of two fermions in the same single-particle state and such a state is not possible.

The completely symmetric wavefunction (ignoring the spin) for two bosons in which one boson is in the single-particle state $\psi_{n_{1}}$ and the other boson is in the single-particle state $\psi_{n_{2}}\left(n_{1} \neq n_{2}\right)$ is $\Psi\left(x_{1}, x_{2}\right)=$ $\frac{1}{\sqrt{2}}\left[\psi_{n_{1}}\left(x_{1}\right) \psi_{n_{2}}\left(x_{2}\right)+\psi_{n_{2}}\left(x_{1}\right) \psi_{n_{1}}\left(x_{2}\right)\right]$. The two bosons can be in the same single-particle state. For example, the completely symmetric wavefunction for two bosons in the single-particle state $\psi_{n_{1}}$ is $\Psi\left(x_{1}, x_{2}\right)=\psi_{n_{1}}\left(x_{1}\right) \psi_{n_{1}}\left(x_{2}\right)$.

When considering the spin part of the wavefunction for a 
single-particle, we will use the notation $\left|s_{i}, m_{s_{i}}\right\rangle$, in which $s_{i}$ and $m_{s_{i}}$ are the quantum numbers corresponding to the total spin and z-component of the spin for the $i^{t h}$ particle, respectively. The states $\left|s_{1}, m_{s_{1}}\right\rangle$ are eigenstates of $\hat{S}_{1}^{2}$ and $\hat{S}_{1 z}$ and the states $\left|s_{2}, m_{s_{2}}\right\rangle$ are eigenstates of $\hat{S}_{2}^{2}$ and $\hat{S}_{2 z}$. We will use the following abbreviated notation for a spin-1/2 particle: $|\uparrow\rangle_{1}=\left|s_{1}, m_{s_{1}}\right\rangle=|1 / 2,1 / 2\rangle_{1}$ and $|\downarrow\rangle_{1}=$ $\left|s_{1}, m_{s_{1}}\right\rangle=|1 / 2,-1 / 2\rangle_{1}$ for electron 1 in the "spin up" and "spin down" state, respectively, and $|\uparrow\rangle_{2}=\left|s_{2}, m_{s_{2}}\right\rangle=$ $|1 / 2,1 / 2\rangle_{2}$, and $|\downarrow\rangle_{2}=\left|s_{2}, m_{s_{2}}\right\rangle=|1 / 2,-1 / 2\rangle_{2}$ for electron 2 in the "spin up" and "spin down" state, respectively.

When considering the spin part of the wavefunction for the two spin-1/2 particles in the "uncoupled" representation in the product space, we will use the notation $|\uparrow \uparrow\rangle=\mid \uparrow$ \rangle$_{1}|\uparrow\rangle_{2},|\uparrow \downarrow\rangle=|\uparrow\rangle_{1}|\downarrow\rangle_{2},|\downarrow \uparrow\rangle=|\downarrow\rangle_{1}|\uparrow\rangle_{2}$, and $|\downarrow \downarrow\rangle=|\downarrow\rangle_{1}|\downarrow\rangle_{2}$ for the basis states. We will also use the notation in the "coupled" representation $\left|s, m_{s}\right\rangle$ (in which the quantum numbers $s$ and $m_{s}$ correspond to the total spin and the $z$ component of the total spin, respectively). The states $\left|s, m_{s}\right\rangle$ in the coupled representation are eigenstates of $\hat{S}^{2}$ and $\hat{S}_{z}$ where $\vec{S}=\vec{S}_{1}+\vec{S}_{2}$. For a system of two spin$1 / 2$ particles $\left(s_{1}=1 / 2, s_{2}=1 / 2\right)$, the quantum number $s=s_{1}+s_{2}=1 / 2+1 / 2=1$ or $s=\left|s_{1}-s_{2}\right|=$ $|1 / 2-1 / 2|=0$. If the total spin quantum number is $s=1$ then the corresponding $m_{s}=-1,0,1$ or if $s=0$ then the corresponding $m_{s}=0$, so that states in the coupled representation are given by $\{|1,1\rangle,|1,0\rangle,|1,-1\rangle,|0,0\rangle\}$. We will use the following abbreviated notation for a complete set of normalized states for a system of two spin-1/2 particles in the coupled representation $\left|s, m_{s}\right\rangle$ written in terms of states in the uncoupled representation $\left(\left|s_{1}, m_{s_{1}}\right\rangle\left|s_{2}, m_{s_{2}}\right\rangle\right)$ :

$$
\begin{aligned}
& \begin{array}{lll}
|1,1\rangle= & |\uparrow\rangle_{1}|\uparrow\rangle_{2} & =|\uparrow \uparrow\rangle \\
|1,-1\rangle= & |\downarrow\rangle_{1}|\downarrow\rangle_{2} & =|\downarrow\rangle
\end{array} \\
& |1,-1\rangle=|\downarrow\rangle_{1}|\downarrow\rangle_{2} \quad=|\downarrow \downarrow\rangle \\
& |1,0\rangle=\frac{1}{\sqrt{2}}\left(|\uparrow\rangle_{1}|\downarrow\rangle_{2}+|\downarrow\rangle_{1}|\uparrow\rangle_{2}\right)=\frac{1}{\sqrt{2}}(|\uparrow \downarrow\rangle+|\downarrow \uparrow\rangle) \\
& |0,0\rangle=\frac{1}{\sqrt{2}}\left(|\uparrow\rangle_{1}|\downarrow\rangle_{2}-|\downarrow\rangle_{1}|\uparrow\rangle_{2}\right)=\frac{1}{\sqrt{2}}(|\uparrow \downarrow\rangle-|\downarrow \uparrow\rangle) \text {. }
\end{aligned}
$$

One feature of the coupled states for two spin-1/2 particles is that they are either completely symmetric or completely antisymmetric with respect to exchange of the two particles. For example, $|\uparrow \uparrow\rangle,|\downarrow \downarrow\rangle, \frac{1}{\sqrt{2}}(|\uparrow \downarrow\rangle+|\downarrow \uparrow\rangle)$ are completely symmetric spin states for two fermions. It is important to note that any linear combination of these three symmetric spin states is also a completely symmetric spin state (i.e., $C_{1} \mid \uparrow \uparrow$ \rangle$+C_{2}|\downarrow \downarrow\rangle+\frac{C_{3}}{\sqrt{2}}(|\uparrow \downarrow\rangle+|\downarrow \uparrow\rangle)$ is a completely symmetric spin state in which $C_{1}, C_{2}$, and $C_{3}$ are constants such that $\left.\left|C_{1}\right|^{2}+\left|C_{2}\right|^{2}+\left|C_{3}\right|^{2}=1\right)$. The state $\frac{1}{\sqrt{2}}(|\uparrow \downarrow\rangle-|\downarrow \uparrow\rangle)$ is the completely antisymmetric spin state of the two fermions.

\section{METHODOLOGY}

Student difficulties with fundamental concepts involving a system of $N$ identical particles were first investigated using three years of data involving responses to openended and multiple-choice questions administered after traditional instruction in relevant concepts from 57 upperlevel undergraduate students in a junior/senior level QM course and 30 graduate students in the second semester of the graduate core QM course at a large research university. Additional insight was gained concerning these difficulties from responses of 14 students during a total of 81 hours of individual interviews. The interviewed students were a subset of the 57 undergraduate and 30 graduate students who volunteered to be interviewed. A "think aloud" protocol was used during the interviews in which students were asked to think aloud as they answered the questions posed without being disturbed. Once the students had answered each question to the best of their ability, we asked them to clarify their reasoning and probed deeper into the origin of the difficulties.

In order to familiarize the students with the notation, students were given that the spatial wavefunction for a system of two non-interacting identical particles has terms such as $\psi_{n_{1}}\left(x_{1}\right) \psi_{n_{2}}\left(x_{2}\right)$ since these are possible basis states for a two-particle system, where $\psi_{n_{1}}\left(x_{1}\right)$ and $\psi_{n_{2}}\left(x_{2}\right)$ are the single-particle wavefunctions for particles in states $n_{1}$ and $n_{2}$ and with coordinates $x_{1}$ and $x_{2}$, respectively.

Below, we discuss difficulties in response to questions that were posed either as in-class clicker questions, open-ended questions on quizzes or exams, or in individual interviews.

\section{STUDENT DIFFICULTIES}

A. Difficulty realizing that the Hamiltonian for a system of $N$ non-interacting identical particles should be written in terms of the sum of the single-particle Hamiltonians in the product-space:

To investigate students' understanding of the Hamiltonian for a system of $N$ non-interacting particles, question Q1 was asked after traditional instruction.

Q1. Write the Hamiltonian for a system of $N$ non-interacting identical particles in terms of the Hamiltonian for the $i^{\text {th }}$ particle $(i=1,2, \ldots, N)$.

Below, we discuss two types of common difficulties students had with the many-particle Hamiltonian for a system of $N$ non-interacting identical particles.

Some students incorrectly wrote the many-particle Hamiltonian as the sum of the single-particle Hamiltonians $\hat{H}_{i}$ in which $\hat{H}_{i}$ is in the Hilbert space of individual particles. The Hamiltonian for a system of $N$ non-interacting identical particles is the sum of the $M^{N}$-dimensional individual Hamiltonians $\hat{\mathbf{H}}_{i}$ for each particle $i$. Interviews suggest that many students struggled to correctly reason about the dimension of the many-particle Hamiltonian. In Q1, some students incorrectly claimed that the Hamiltonian for the system of $N$ non-interacting identical particles was $\sum \hat{H}_{i}$ (as opposed to $\sum \hat{\mathbf{H}}_{i}$ ). These students did not realize that each term in the sum must be an element in the $M^{N}$ dimensional product space (instead of an $M$-dimensional Hilbert space). They had difficulty identifying that the singleparticle Hamiltonian $\hat{H}_{i}$ only acts on the subspace of the $i^{t h}$ particle and that the many-particle Hamiltonian $\hat{\mathbf{H}}_{i}$ must have the dimensionality of the product space and contain identity operators in the subspaces of the $N-1$ other particles as 
discussed in Section I. For example, the Hamiltonian $\hat{H}_{i}$ for each spin-1/2 particle can be represented as a $2 \times 2$ matrix in a given basis and the Hamiltonian for a system of two identical spin-1/2 particles should be represented as a $4 \times 4$ matrix in the product space. However, students with this type of difficulty often claimed that the Hamiltonian for the two noninteracting spin- $1 / 2$ particles is a $2 \times 2$ matrix resulting from adding the two $2 \times 2$ matrices for each spin-1/2 particle since the particles are non-interacting. This type of difficulty in determining the dimensionality of an operator in the product space has been documented in other contexts [25].

Other students incorrectly claimed that the answer to Q1 was that the Hamiltonian for non-interacting particles was the direct product of the $M^{N}$-dimensional single-particle Hamiltonians. Some students incorrectly claimed that the many-particle Hamiltonian was $\hat{\mathbf{H}}=$ $\hat{\mathbf{H}}_{1} \otimes \hat{\mathbf{H}}_{2} \otimes \hat{\mathbf{H}}_{3} \otimes \ldots \otimes \hat{\mathbf{H}}_{N}$. Students with this type of difficulty did not realize that each single-particle Hamiltonian $\hat{\mathbf{H}}_{i}$ is $M^{N}$-dimensional, and that the direct product produces a $\left(M^{N}\right)^{N}$-dimensional many-particle Hamiltonian. Other students incorrectly claimed that the many-particle Hamiltonian for the non-interacting particles is $\hat{\mathbf{H}}=$ $\hat{H}_{1} \otimes \hat{H}_{2} \otimes \hat{H}_{3} \otimes \ldots \otimes \hat{H}_{N}$. Students with this type of response did not realize that for a system of non-interacting identical particles, there is no entanglement between the single particle Hamiltonians $\hat{H}_{i}$. One interviewed student stated that "since the wavefunction of the many-particle system is the product of the single-particle wavefunctions, then the Hamiltonian for the system of $N$ non-interacting identical particles is also a product of the individual Hamiltonians." This student correctly claimed that the many-particle wavefunction for non-interacting particles is written in terms of the direct product of the single-particle wavefunctions, but incorrectly inferred that the Hamiltonian for the many-particle system is also written as the direct product (as opposed to the sum) of the individual singleparticle Hamiltonians $\hat{\mathbf{H}}_{\mathbf{i}}$.

B. Difficulty realizing that each indistinguishable particle should have its own unique coordinate: Many students had difficulty with the fact that each quantum particle in a system of identical particles is indistinguishable but each particle is still expressed with a unique coordinate. For example, for the many-particle wavefunction for a system of $N$ indistinguishable particles, the coordinates $x_{1}, x_{2}, x_{3}, \ldots, x_{N}$ can be used to label the particles. The symmetrization requirement of the many-particle wavefunction ensures indistinguishability and accounts for the fact that there is no way to determine which particle is in which single-particle state. To probe whether students are able to identify that each identical particle has a unique coordinate, question Q2 was posed after traditional lecturebased instruction:

Q2. For a system of three non-interacting identical particles, write a three-particle wavefunction in position representation where all three particles are in different single-particle states for the following three cases:
TABLE I. The percentage of undergraduate $(\mathrm{N}=25)$ and graduate students $(\mathrm{N}=30)$ who displayed the given difficulty in $\mathrm{Q} 2$ after traditional lecture-based instruction.

\begin{tabular}{|c|c|c|}
\hline Difficulty & $\begin{array}{c}\text { Undergraduate } \\
\text { Students (\%) }\end{array}$ & $\begin{array}{c}\text { Graduate } \\
\text { Students (\%) }\end{array}$ \\
\hline $\begin{array}{c}\text { Did not use unique coordinates } \\
\text { for each particle }\end{array}$ & 32 & 43 \\
\hline $\begin{array}{c}\text { Wrote the wavefunction as the sum } \\
\text { of the single-particle wavefunctions }\end{array}$ & 16 & 10 \\
\hline
\end{tabular}

indistinguishable fermions, indistinguishable bosons, and identical particles treated as distinguishable. If there is no possible three-particle wavefunction for the given system of three particles, state the reason. Ignore the spin of the particles and only consider the spatial part of the wavefunction.

Regardless of the type of particle, there should be unique coordinates for each identical particle in the three-particle wavefunction. However, many students with this type of difficulty claimed that since there is no way to determine which particle is in which single-particle state, there is no way to assign a distinct coordinate to each indistinguishable particle. For example, one interviewed student incorrectly claimed that "we must use the coordinate $x$ for all the indistinguishable particles since we don't know where each particle is." This student went on to claim that the manyparticle wavefunction for a system of three indistinguishable particles in which one of the particles is in each of the singleparticle stationary states labeled $\psi_{n_{1}}(x), \psi_{n_{2}}(x)$, and $\psi_{n_{3}}(x)$ is $\psi_{n_{1}}(x) \psi_{n_{2}}(x) \psi_{n_{3}}(x)$. Table I summarizes the number of students who displayed this type of difficulty in question Q2 after traditional lecture-based instruction.

C. Difficulty realizing that the many-particle basis states must be written in terms of the product (NOT the SUM) of the single-particle wavefunctions: Many students struggled to write the basis states for the many-particle wavefunction in terms of the product of the single-particle wavefunctions. Question Q2 was intended to probe whether students were able to identify that the wavefunction for a system of identical particles must be expressed in terms of the direct product (as opposed to the sum) of the single-particle wavefunctions, since these form possible basis states for a many-particle system. For example, the three-particle wavefunction for a system of three fermions $\left(n_{1} \neq n_{2} \neq n_{3}\right)$ is $\frac{1}{\sqrt{6}}\left[\psi_{n_{1}}\left(x_{1}\right) \psi_{n_{2}}\left(x_{2}\right) \psi_{n_{3}}\left(x_{3}\right)-\psi_{n_{1}}\left(x_{1}\right) \psi_{n_{3}}\left(x_{2}\right) \psi_{n_{2}}\left(x_{3}\right)-\right.$ $\psi_{n_{2}}\left(x_{1}\right) \psi_{n_{1}}\left(x_{2}\right) \psi_{n_{3}}\left(x_{3}\right)+\psi_{n_{2}}\left(x_{1}\right) \psi_{n_{3}}\left(x_{2}\right) \psi_{n_{1}}\left(x_{3}\right)+$ $\left.\psi_{n_{3}}\left(x_{1}\right) \psi_{n_{1}}\left(x_{2}\right) \psi_{n_{2}}\left(x_{3}\right)-\psi_{n_{3}}\left(x_{1}\right) \psi_{n_{2}}\left(x_{2}\right) \psi_{n_{1}}\left(x_{3}\right)\right]$. In order to satisfy the symmetrization requirements, the threeparticle wavefunction for a system of three fermions consists of six terms. However, each term is expressed as the direct product of the single-particle wavefunctions.

Some students with this type of difficulty incorrectly expressed the many-particle wavefunction in question Q2 in terms of the sum (as opposed to the direct product) 
of the single-particle wavefunctions, such as $\psi_{n_{1}}\left(x_{1}\right)+$ $\psi_{n_{2}}\left(x_{2}\right)+\psi_{n_{3}}\left(x_{3}\right)$. For example, one interviewed student incorrectly stated that "since the particles do not interact, the wavefunction will just be the sum of the (singleparticle) wavefunctions." This student was later asked whether the wavefunction for a system of identical particles was always expressed as the sum he had written down or whether there were any situations in which the manyparticle wavefunction could be written in terms of the direct product of the single-particle wavefunctions and he stated that only "if the particles were interacting, then the manyparticle wavefunction could be expressed as the product (of the single-particle) wavefunctions." However, regardless of whether the particles are interacting or non-interacting, the many-particle wavefunction can always be expressed in terms of the direct product of the single-particle wavefunction since the many-particle wavefunctions obtained from the product of the single-particle wavefunctions form a basis for the Hilbert space for a many-particle system. Table I shows the percentages of students who expressed the many-particle wavefunction as a sum of the single-particle wavefunctions (as opposed to terms involving the product of the singleparticle wavefunctions) in response to question Q2.

D. Incorrectly determining the symmetry based on the appearance of a $+/-$ sign in the many-particle wavefunction: In order to recognize and generate a manyparticle wavefunction for a system of indistinguishable particles, students must be able to determine a completely symmetric/antisymmetric wavefunction involving both spatial and spin degrees of freedom. Question Q3 was posed during the think aloud interview to investigate students' proficiency at identifying whether the spin part of a wavefunction was symmetric or antisymmetric. Students were given Eq. (1) and were familiar with the shorthand notation used in Q3.

Q3. For the spin part of the wavefunction of a twoparticle system given below, identify whether the spin state is symmetric, antisymmetric, or neither symmetric nor antisymmetric with respect to exchange of the two particles. Explain your reasoning. (a) $|\uparrow \uparrow\rangle$, (b) $|\downarrow \downarrow\rangle$, (c) $|\uparrow \downarrow\rangle$, (d) $\frac{1}{\sqrt{2}}(|\uparrow \downarrow\rangle+|\downarrow \uparrow\rangle)$, (e) $\frac{1}{\sqrt{2}}(|\uparrow \downarrow\rangle-|\downarrow \uparrow\rangle)$

In Q3, options (a), (b), and (d) are symmetric spin states (triplet states) since exchanging the two particles results in the same wavefunction. Option (e) in question Q3 is an antisymmetric spin state (singlet state) since exchanging the two particles results in the original wavefunction multiplied by -1 . Option (c) in question Q3 is neither a symmetric nor an antisymmetric spin state.

Some students incorrectly applied a heuristic by which they claimed that a wavefunction is symmetric if the wavefunction is written in terms of a sum. These students simply looked for all "+" signs to determine that a wavefunction is symmetric. They claimed that any wavefunction written as terms added together is a symmetric wavefunction. By a similar logic, these same students looked for a "-" sign to determine whether a given wavefunction is antisymmetric. Some claimed that any wavefunction that has at least one negative sign is antisymmetric. In particular, their determination of whether the wavefunction is antisymmetric did not depend on the complete symmetrization requirement. They merely looked for the presence of at least one minus sign in the wavefunction to determine that the wavefunction is antisymmetric. For example, in response to question Q3(a) and (b), one interviewed student incorrectly claimed that the spin state given by $|\uparrow \uparrow\rangle$ or $|\downarrow \downarrow\rangle$ is neither symmetric nor antisymmetric as "the wavefunction is not a sum so it can't be symmetric and there is not a minus sign, so it can't be antisymmetric." However, the spin state given by $|\uparrow \uparrow\rangle$ or $|\downarrow \downarrow\rangle$ is completely symmetric as the exchange of the two particles results in the same state. Thus, there need not be a plus sign in order for a wavefunction to be symmetric. Other students with this type of reasoning also used similar types of reasoning when determining the symmetry of a many-particle wavefunction.

\section{SUMMARY AND FUTURE PLAN}

Our investigation suggests that upper-level undergraduate and graduate students have many common difficulties with fundamental concepts involving a system of non-interacting identical particles. Since the paradigm of QM is novel, providing appropriate scaffolding support becomes critical and research on student difficulties must be used as a guide in developing curricula and pedagogies to help students develop a solid grasp of these concepts. We are using the difficulties found in this context as a guide to develop and validate a Quantum Interactive Learning Tutorial (QuILT) that strives to provide appropriate guidance and scaffolding support to help students develop a good grasp of these concepts. The QuILT focuses on helping students develop a coherent understanding of fundamental concepts for a system of non-interacting identical particles, e.g., the form of the Hamiltonian and the many-particle stationary state wavefunction for a system of identical particles and how to generate many-particle stationary state wavefunctions that satisfy a given symmetrization requirement. It helps students learn that the Hamiltonian for a system of noninteracting identical particles can be expressed as a sum in the product space and the many-particle stationary state wavefunction is expressed in terms of the sum of direct products of the single particle stationary state wavefunctions in which each indistinguishable particle should have its own unique coordinate. The QuILT also helps students determine the completely symmetric/antisymmetric manyparticle stationary state wavefunction by using all the permutations of the labels for the states or the coordinates in the products of the single-particle states with the appropriate symmetrization requirement [46]. Further details about the development and validation of the QuILT will be discussed in future publications.

\section{ACKNOWLEDGEMENTS}

We thank the NSF for award PHY-1806691. 
[1] C. Singh, Student understanding of quantum mechanics, Am. J. Phys. 69, 885 (2001).

[2] R. Muller and H. Wiesner Teaching quantum mechanics on an introductory level, Am. J. Phys. 70, 200 (2002).

[3] D. Zollman, S. Rebello, and K. Hogg, Quantum mechanics for everyone: Hands-on activities integrated with technology, Am. J. Phys. 70, 252 (2002).

[4] M. Wittmann et al., Investigating student understanding of quantum physics: Spontaneous models of conductivity, Am. J. Phys. 70, 218 (2002).

[5] C. Singh, M. Belloni, and W. Christian, Improving students' understanding of quantum mechanics, Physics Today 59 (8), 43 (2006).

[6] C. Singh, Transfer of learning in quantum mechanics, in Proc. Phys. Educ. Res. Conf. 790 p. 23 (2005).

[7] C. Singh, Assessing and improving student understanding of quantum mechanics, in Proc. Phys. Educ. Res. Conf. 818, p. 69 (2006).

[8] C. Singh, Student difficulties with quantum mechanics formalism, in Proc. Phys. Educ. Res. Conf. 883, p. 185 (2007).

[9] C. Singh, Helping students learn quantum mechanics for quantum computing, in Proc. Phys. Educ. Res. Conf. 883 p. 42 AIP Conf. Proc. (2007).

[10] C. Singh, Interactive learning tutorials on quantum mechanics, Am. J. Phys. 76, 400 (2008).

[11] D. McIntyre, J. Tate and C. Manogue, Integrating computational activities into the upper-level Paradigms in Physics curriculum at Oregon State University, Am. J. Phys. 76, 340 (2008).

[12] C. Singh, Student understanding of quantum mechanics at the beginning of graduate instruction, Am. J. Phys. 76, 277 (2008).

[13] C. Singh and G. Zhu, Cognitive issues in learning advanced physics: An example from quantum mechanics, in Proc. Phys. Educ. Res. Conf. 1179, p. 63 (2009).

[14] A. Kohnle et al., Developing and evaluating animations for teaching quantum mechanics concepts, Eur. J. Phys. 31, 1441 (2010).

[15] A. J. Mason and C. Singh, Do advanced students learn from their mistakes without explicit intervention?, Am. J. Phys. 78, 760 (2010).

[16] S. Y. Lin and C. Singh, Categorization of quantum mechanics problems by professors and students, Euro. J. Phys. 31, 57(2010).

[17] G. Zhu and C. Singh, Improving students' understanding of quantum mechanics via the Stern-Gerlach experiment, Am. J. Phys. 79, 499 (2011).

[18] C. Singh and G. Zhu, Improving students' understanding of quantum mechanics by using peer instruction tools, in Proc. Phys. Educ. Res. Conf. 1413, p. 77 (2012).

[19] G. Zhu and C. Singh, Surveying students' understanding of quantum mechanics in one spatial dimension, Am. J. Phys. 80, 252 (2012).

[20] G. Zhu and C. Singh, Improving students' understanding of quantum measurement: I. Investigation of difficulties Phys. Rev. ST PER 8, 010117 (2012).

[21] G. Zhu and C. Singh, Improving students' understanding of quantum measurement: II. Development of research-based learning tools, Phys. Rev. ST PER 8, 010118 (2012).
[22] G. Zhu and C. Singh, Improving student understanding of addition of angular momentum in quantum mechanics, Phys. Rev. ST PER 9, 010101 (2013).

[23] A. Kohnle et al., New introductory quantum mechanics curriculum, Eur. J. Phys. 35, 015001 (2014).

[24] E. Marshman and C. Singh, Framework for understanding student difficulties in quantum mechanics, Phys. Rev. ST PER 11, 020119 (2015).

[25] C. Singh and E. Marshman, Review of student difficulties in quantum mechanics, Phys. Rev. ST PER 11, 020117 (2015).

[26] E. Gire et al., The structural features of algebraic quantum notations, Phys. Rev. ST PER 11, 020109 (2015).

[27] P. Emigh et al., Student understanding of time dependence in quantum mechanics, Phys. Rev. ST Phys. Educ. Res. 11, 020112 (2015).

[28] G. Passante et al., Examining student ideas about energy measurements on quantum states across undergraduate and graduate levels, Phys. Rev. ST PER 11, 020111 (2015).

[29] B. Brown, A. Mason, and C. Singh, Improving performance in quantum mechanics with explicit incentives to correct mistakes, Phys. Rev. PER 12, 010121 (2016).

[30] E. Marshman and C. Singh, Interactive tutorial to improve student understanding of single photon experiments involving a Mach-Zehnder interferometer, Eur. J. Phys. 37, 024001 (2016).

[31] E. Marshman and C. Singh, Investigating and improving student understanding of the probability distributions for measuring physical observables in quantum mechanics, Eur. J. Phys. 38, 025705 (2017).

[32] E. Marshman and C. Singh, Investigating and improving student understanding of quantum mechanics in the context of single photon interference, Phys. Rev. PER 13, 010117 (2017).

[33] E. Marshman and C. Singh, Investigating and improving student understanding of quantum mechanical observables and their corresponding operators in Dirac notation, Eur. J. Phys. 39, 015707 (2017).

[34] S. Siddiqui and C. Singh, How diverse are physics instructors? attitudes and approaches to teaching undergraduate-level quantum mechanics? Eur. J. Phys. 38, 035703 (2017).

[35] A. Maries, R. Sayer and C. Singh, Effectiveness of interactive tutorials in promoting "which-path" information reasoning in advanced quantum mechanics, Phys. Rev. PER 13, 020115 (2017).

[36] R. Sayer, A. Maries and C. Singh, A quantum interactive learning tutorial on the double-slit experiment to improve student understanding of quantum mechanics," Phys Rev PER 13, 010123 (2017).

[37] V. Dini and D. Hammer, Case study of a successful learner's epistemological framings of quantum mechanics, Phys. Rev. Phys. Educ. Res. 13, 010124 (2017).

[38] C. Keebaugh, E. Marshman and C. Singh, Investigating and addressing student difficulties with a good basis for finding perturbative corrections in the context of degenerate perturbation theory Eur. J. Phys. 39, 055701 (2018).

[39] E. Marshman and C. Singh, Investigating and improving student understanding of the expectation values of observables in quantum mechanics, Eur. J. Phys. 38, 045701 (2017).

[40] C. Keebaugh, E. Marshman and C. Singh, Investigating and addressing student difficulties with the corrections to the energies of the hydrogen atom for the strong and weak field 
Zeeman effect, Eur. J. Phys. 39, 045701 (2018).

[41] C. Keebaugh, E. Marshman and C. Singh, Improving student understanding of fine structure corrections to the energy spectrum of the hydrogen atom, Am. J. Phys. 87, 594 (2019).

[42] C. Keebaugh, E. Marshman and C. Singh, Improving student understanding of a system of identical particles with a fixed total energy, Am. J. Phys. 87, 583 (2019)
[43] C. Keebaugh, E. Marshman and C. Singh, Improving student understanding of corrections to the energy spectrum of the hydrogen atom for the Zeeman effect, Phys. Rev. Phys. Educ. Res. 15, 010113 (2019).

[44] E. Marshman and C. Singh, Validation and administration of a conceptual survey on the formalism and postulates of quantum mechanics, Phys. Rev. PER 15, 020128 (2019).

[45] S. Devore and C. Singh, An interactive learning tutorial on quantum key distribution, Phys. Rev. PER 16, 010126 (2020).

[46] https://www.physport.org/curricula/quilts/ 